\title{
PARÂMETROS QUANTITATIVOS DA ANATOMIA DA MADEIRA DE EUCALÍPTO QUE CRESCEU EM DIFERENTES LOCAIS ${ }^{1}$
}

José Geraldo Lima de Oliveira², José Tarcísio da Silva Oliveira³ ${ }^{3}$ Júpiter Israel Muro Abad ${ }^{4}$, Aderbal Gomes da Silva ${ }^{5}$, Nilton César Fiedler ${ }^{6}$ e Graziela Baptista Vidaure ${ }^{7}$

\begin{abstract}
RESUMO - Sabe-se da existência de influência do local de crescimento nas propriedades da madeira e que estas são influenciadas principalmente pela sua estrutura anatômica. Este trabalho teve como objetivo avaliar parâmetros quantitativos da estrutura anatômica da madeira de eucalipto de um clone de híbrido natural de Eucalyptus grandis W. Hill ex-Maiden, ocorrentes na localidade de Rio Claro-SP, com 64 meses de idade. As 138 árvores foram cultivadas nos os municípios de Aracruz, Domingos Martins, Alto Rio Novo e São Mateus - ES e Mutum e Aimorés - MG. Os parâmetros anatômicos mensurados foram o diâmetro e a frequência vascular, o comprimento, a largura, o diâmetro do lume e a espessura da parede das fibras e ainda a altura, largura e frequência de raios. De acordo com os resultados obtidos pode-se concluir que relativo aos vasos houve diferenças significativas entre as regiões. Quanto aos raios, também foram verificadas diferenças significativas relativas à altura, largura e frequência diversas regiões. As madeiras produzidas na região de Aracruz possuem fibras de comprimento e espessura de paredes muito inferiores às produzidas nas demais regiões estudadas.
\end{abstract}

Palavras-chave: Madeira de eucalipto, Clone e Estrutura anatômica.

\section{ANATOMICAL STRUCTURE MEASUREMENTS IN EUCALYPT WOOD THAT GROWN IN DIFFERENTS PLACES}

\begin{abstract}
It is known the influence of the growth place in the properties of the woods and that they are affected mainly by the anatomical structures. The objective of this work was to evaluate the quantitative parameters of the anatomical structure of the eucalypt wood of one clone of the natural hybrid of Eucalyptus grandis at 64 months of age occuring in Rio Claro, state of São Paulo. The 138 trees were grown in Aracruz, Domingos Martins, Alto Rio Novo, São Mateus in the state of Espírito Santo and in Mutum and Aimorés in the state of Minas Gerais. The anatomical parameters measured were diameter and vessel frequency, length, fiber and diameter of the fiber, fiber wall thickness and width, height and frequency of the rays. According to the results, it can be concluded that, in relation to the vessels, there were significant differences among all regions. It was also observed significant differences in the parameters related to the rays in all regions. The wood produced in the Aracruz region had fibers with length and wall thickness with a much lower quality than the wood in the other studied regions.
\end{abstract}

Keywords: Eucalypt wood, Clone and Anatomicals properties.

\footnotetext{
${ }^{1}$ Recebido em 08.05.2009 e aceito para publicação em 19.04.2012.

2 Departamento de Ciências Florestais e da Madeira, Universidade Federal do Espírito Santo, UFES, Brasil. E-mail: <jjosegeraldolo@yahoo.com.br>.

${ }^{3}$ Departamento de Ciências Florestais e da Madeira, Universidade Federal do Espírito Santo, UFES, Brasil. E-mail: <jjosegeraldolo@yahoo.com.br>.

${ }^{4}$ Centro de Tecnologia - Fibria <jmuro@fibria.com.br>.

${ }^{5}$ Departamento de Ciências Florestais e da Madeira, Universidade Federal do Espírito Santo, UFES, Brasil. E-mail: $<$ aderbalsilva@yahoo.com.br>.

${ }^{6}$ Departamento de Ciências Florestais e da Madeira, Universidade Federal do Espírito Santo, UFES, Brasil. E-mail: <fiedler@pq.cnpq.br>.

${ }^{7}$ Departamento de Ciências Florestais e da Madeira, Universidade Federal do Espírito Santo, UFES, Brasil. E-mail: $<$ grazividaurre@gmail.com>.
} 


\section{INTRODUÇÃO}

O gênero Eucalyptus tem-se destacado nos últimos anos pela grande diversidade de espécies botânicas e boa adaptação aos mais diferentes tipos de ambiente. A adoção de práticas sustentáveis de manejo florestal, objeto de uma das grandes realizações da pesquisa florestal no Brasil, proporcionou o desenvolvimento de uma tecnologia silvicultural de florestas plantadas reconhecida no mundo todo. A aplicação dessa tecnologia, bem como o grau de melhoramento genético dessas espécies, é fundamental na agregação da qualidade e valor ao produto final industrializado.

A caracterização anatômica da madeira proporciona seu melhor conhecimento e direciona de forma mais segura e adequada sua utilização para os diversos fins.

A utilização de clones poderá, de modo geral, levar à produção de madeira de qualidade e em grande escala, possibilitando a seleção prévia de características que poderão assegurar ganhos de qualidade ao produto final.

Barrichelo e Brito (1976) descreveram as características anatômicas da madeira de espécies do gênero Eucalyptus, observando que o comprimento das fibras variou entre 750,00 e 1.300,00 $\mu \mathrm{m}$, com a média próxima de 1.000,00 $\mu \mathrm{m}$. Quanto ao diâmetro das fibras, a variação ficou entre 15,00 e 20,00 $\mu \mathrm{m}$. O diâmetro dos vasos variou de 50,00 a 300,00 $\mu \mathrm{m}$ e a frequência, entre 5 e 100/ $\mathrm{mm}^{2}$. Ainda de acordo com esses autores, os percentuais de fibras, vasos e parênquima no tecido do lenho foram 65,17 e $18 \%$, respectivamente.

Muitos autores têm analisado os parâmetros anatômicos da madeira de espécies do gênero Eucalyptus com os mais diversos objetivos e levando em consideração a posição da amostra no caule, a idade das plantas e o ambiente onde ocorrem. Oliveira (1998) estudou as características tecnológicas da madeira de sete espécies de Eucalyptus com idade média de 17 anos para a construção civil. Ele concluiu que, apesar de o lenho dessa madeira ser bastante homogêneo sob o aspecto morfológico da constituição anatômica, observou considerável variação quanto às dimensões e frequência de ocorrência desses constituintes anatômicos. Esse mesmo autor ainda afirmou que, do ponto de vista tecnológico, relativo à influência nas demais propriedades da madeira e, consequentemente, da sua utilização final, torna-se relevante o maior destaque dado aos estudos quantitativos, em relação aos meramente descritivos.
Gonçalves et al. (2004), através de vasta revisão bibliográfica sobre os efeitos de tratamentos silviculturais (fertilização, espaçamento, controle de ervas daninhas e desbastes) na produtividade e qualidade da madeira em plantações de eucalipto, afirmaram não haver regra específica quanto aos diferentes tratamentos silviculturais que afetam a densidade da madeira, mas fatores ambientais como disponibilidade de luz, pluviosidade e temperatura causam efeitos mais significativos na formação da madeira.

Ramírez e Rodríguez (2009), estudando a variação na direção medula-casca de alguns parâmetros anatômicos da madeira de clones de Eucalyptus globulus no Chile, encontraram comportamento diferenciado dos diversos elementos anatômicos analisados. De acordo com esses autores, entre clones da mesma espécie foram encontradas grandes variações para frequência e área coberta por vasos, ao passo que pequenas variações foram observadas na espessura de parede, largura e diâmetro de lume das fibras. Na direção medula-casca, a área ocupada por vasos aumentou gradualmente, tendo decrescido a frequência desses elementos. Ainda de acordo com Ramírez e Rodríguez (2009), a espessura de parede das fibras não diferiu significativamente da medula para a casca, com os diâmetros da fibra e lume mostrando padrão radial idêntico, ou seja, aumentando da medula para a parte central do raio, até atingirem os valores máximos próximos da casca. Bamber e Humphrey (1963), ao estudarem a madeira de indivíduos de Eucalyptus grandis, relataram grandes diferenças no diâmetro dos vasos, em diferentes posições dos anéis de crescimento no sentido medulacasca e no diâmetro dos vasos, que aumentou de 64,00 ìm no lenho inicial para 141,00 ìm no lenho tardio. Já Bamber et al. (1982) relataram que árvores de eucalipto de rápido crescimento têm menor frequência de vasos em relação ao normal, ou seja, valor de 14,10 poros $/ \mathrm{mm}^{2}$ na madeira normal e 19 poros $/ \mathrm{mm}^{2}$ na madeira de rápido crescimento, induzido por técnicas silviculturais, o que explica a tendência da maior densidade de madeira de árvores de rápido crescimento, embora não significativa. Quanto ao diâmetro dos vasos, também houve diferença significativa entre o valor de 61,00 ìm para o lenho de rápido crescimento e de 74,00 ìm para o de crescimento lento.

Revista Árvore, Viçosa-MG, v.36, n.3, p.559-567, 2012

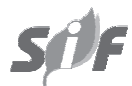


Bamber e Humphrey (1963), estudando a madeira de árvores de Eucalyptus grandis, relataram grandes diferenças no diâmetro dos vasos, em diferentes posições dos anéis de crescimento no sentido medula-casca. $\mathrm{O}$ diâmetro dos vasos aumentou de 64,00 ìm no lenho inicial para 141,00 ìm no tardio. Bamber et al. (1982) relataram que árvores de eucalipto de rápido crescimento têm menor frequência de vasos em relação às árvores de crescimento normal $\left(14,10 / \mathrm{mm}^{2}\right)$, explicando a tendência da maior densidade da madeira de árvores de rápido crescimento, embora não significativa. Quanto ao diâmetro dos vasos, este foi expressivo entre árvores de crescimentos rápido e normal, com valores variando entre 61,00 e 74,00 ìm.

Autores como Rodrigues et al. (1986), Pires e Parente (1986) e Drumond et al. (1998) afirmaram que, entre a maioria das espécies de eucalipto introduzidas no Brasil, o Eucalyptus urophylla é a que apresenta maior estabilidade genética em todas as áreas onde foi testada, considerando-se como uma das espécies de maior potencial para reflorestamento devido ao seu bom crescimento em quase todo o Brasil.

Silva et al. (2007), estudando a influência da idade e posição radial nas dimensões das fibras e dos vasos da madeira de Eucalyptus grandis, verificaram que tais dimensões apresentaram variações tanto em relação à idade quanto ao sentido medula-casca, com o comprimento, a espessura da parede das fibras e o diâmetro dos vasos mostrando-se positivamente correlacionados com a idade e com a posição radial no sentido medula-casca; a largura e diâmetro do lume das fibras correlacionaram-se negativamente com a idade, mas positivamente com a posição radial, no sentido medula-casca.

Tomazello Filho (1983), estudando as espécies de Eucalyptus globulus e Eucalyptus grandis, ambas com a idade de 10 anos, relatou que a primeira apresentou comprimento de fibras de $0,83 \mathrm{~mm}$ e a segunda, de $1,03 \mathrm{~mm}$.

Bamber (1985) afirmou que as propriedades anatômicas da madeira de eucalipto são muito variáveis e exercem influência tanto no processo de produção quanto nas propriedades do papel. duPlooy(1980) procurou estabelecer relações quantitativas entre as propriedades da madeira de Eucalyptus grandis e da polpa em trabalho realizado na África do Sul. Em seu trabalho, esse autor concluiu que na madeira dessa espécie a espessura da parede celular foi a variável que aparece mais frequente numa equação de regressão múltipla que descreve todas as propriedades da polpa avaliadas. Esse autor afirmou que a resistência ao rasgo, uma das mais importantes propriedades de resistência da polpa, é principal e positivamente correlacionada com a relação entre comprimento e largura da fibra, também chamado de índice de enfeltramento. Outras propriedades importantes são a resistência à tração e a resistência ao estouro, que são dependentes da espessura da parede das fibras, mas ambas negativamente correlacionadas com esse parâmetro.

Wher (1991) informou que as fibras com paredes delgadas combinadas com maiores diâmetros de fibras são encontradas em madeiras de menor massa específica, sendo essas fibras mais suscetíveis ao colapso, favorecendo as ligações entre fibras e também o resultado do refino na fabricação de papel.

Trugilho et al. (2004), estudando a classificação dos clones de Eucalyptus sp para produção de polpa celulósica, verificaram que em 15 clones de Eucalyptus sp de 6 anos de idade, tendo sido coletadas duas árvores por clone, ocorreu grande variação entre eles, e as características que mais contribuíram para essa variação foram o rendimento depurado $(58,10 \%)$ e a viscosidade da polpa celulósica $(35,00 \%)$.

Silva et al. (2007), estudando a influência da idade e da posição radial nas dimensões das fibras e dos vasos da madeira de Eucalyptus grandis, verificaram que tais dimensões apresentaram variações tanto em relação à idade quanto ao sentido medula-casca, com o comprimento, a espessura da parede das fibras e o diâmetro dos vasos mostrando-se positivamente correlacionados com a idade e com a posição radial no sentido medula-casca; a largura e o diâmetro do lume das fibras correlacionaram-se negativamente com a idade, mas positivamente com a posição radial, no sentido medula-casca.

Trugilho et al. (2004), estudando a classificação dos clones de Eucalyptus sp para produção de polpa celulósica, verificaram que em 15 clones de Eucalyptus sp com 6 anos de idade, tendo sido coletadas duas árvores por clone, ocorreu grande variação entre eles, e as características que mais contribuíram para essa variação foram o rendimento depurado $(58,10 \%)$ e a viscosidade da polpa celulósica $(35,00 \%)$.

Este trabalho teve como objetivo avaliar parâmetros quantitativos da estrutura anatômica no que se refere aos vasos, fibras e raios da madeira de eucalipto de um clone de Eucalyptus grandis proveniente de programa de fomento florestal, implantado em regiões dos Estados do Espírito Santo e de Minas Gerais, com idade de 64 meses.

Revista Árvore, Viçosa-MG, v.36, n.3, p.559-567, 2012 


\section{MATERIAL E MÉTODOS}

\subsection{Descrição do material}

Neste estudo foram avaliadas as propriedades anatômicas da madeira de eucalipto cultivada em quatro regiões do Estado do Espírito Santo e duas regiões de Minas Gerais, dentro do programa de fomento florestal da empresa Aracruz Celulose. O material genético é formado por um clone constituído de híbridos naturais de Eucalyptus grandis, originados da localidade de Rio Claro, SP. As árvores utilizadas no estudo possuíam idade de 64 meses.

Foram empregadas amostras em forma de disco, com espessura de aproximadamente $5,0 \mathrm{~cm}$, retirado na altura do DAP, com a utilização de seis árvores de cada uma das localidades das regiões do estudo, que formaram os tratamentos avaliados, apresentados na Tabela 1.

\subsection{Metodologia}

Foram utilizadas amostras de madeira de um clone com 64 meses de idade, procedentes de quatro regiões do Espírito Santo e duas regiões e Minas Gerais, sendo estas as regiões de São Mateus, Aracruz, Domingos Martins e Alto Rio Novo, no Estado do Espírito Santo; e as regiões de Mutum e Aimorés, em Minas Gerais. Com exceção dos povoamentos implantados na região de Aracruz, cujo relevo é plano, todos os demais foram provenientes de áreas inclinadas, com altitudes nas regiões de Aracruz de 49 m, Domingos Martins de 586 m, Mutum de 850 m, São Mateus de 200 m, Aimorés de 850 m e Alto Rio Novo de 750 m. Já em relação à precipitação média anual as regiões apresentaram valores de $1.337,1.109,1.361,883$ e 830 , respectivamente em Aracruz, Mutum, São Mateus, Aimorés e alto Rio Novo. Com relação ao tipo de solo predominante, apenas nas regiões de Domingos Martins e Mutum, com o tipo

Tabela 1 - Relação das regiões e número de árvores avaliadas. Table 1-Regions and number of the assessed trees.

\begin{tabular}{lc}
\hline Região & Número de Árvores \\
\hline Aracruz - ES & 24 \\
Domingos Martins - ES & 24 \\
Mutum - MG & 24 \\
São Mateus - ES & 18 \\
Aimorés - MG & 24 \\
Alto Rio Novo - ES & 24 \\
\hline Total & 138 \\
\hline
\end{tabular}

latossolo; e na região de Aimorés, com o solo do tipo argiloarenoso, esses valores estão disponíveis. De cada uma dessas regiões foram amostradas árvores de localidades distintas, com as regiões de São Mateus sendo compostas por três localidades e as demais, por quatro. De cada localidade foram utilizadas seis árvores, num total de 138 árvores (Tabela 1).

As atividades experimentais foram conduzidas no Laboratório de Ciência da Madeira (LCM) do Departamento de Engenharia Florestal do Centro de Ciências Agrárias da Universidade Federal do Espírito Santo (CCA-UFES).

\subsubsection{Descrição anatômica quantitativa}

Para os estudos anatômicos foram retiradas amostras da região mais externa do cerne, dos respectivos discos. Foram utilizados cortes histológicos e material dissociado do lenho para as mensurações dos parâmetros anatômicos. As análises das características qualitativas do lenho foram feitas ao microscópio de luz (ML) e seguiram as recomendações normativas (COPANT, 1974).

\subsubsection{Mensuração de vasos e raios}

Em todas as amostras do lenho no DAP perfazendo um total de 138 discos, as amostras foram retiradas nas posições de transição do cerne/alburno (cerne periférico). Das amostras foram retirados corpos-deprova com dimensões de 1,0 x 1,5 x 2,0 cm, nas direções radiais, tangenciais e longitudinais, respectivamente. Os parâmetros mensurados relativos aos vasos foram a frequência vascular em poros $/ \mathrm{mm}^{2}$ e o diâmetro tangencial em micrômetro. Para cada disco de madeira foram adotadas 40 repetições, para a frequência e diâmetro tangencial dos vasos.

Com relação aos raios, foram mensuradas a altura (em $\mu \mathrm{m}$ e $\mathrm{n}^{\circ}$ de células), largura (em $\left.\mu \mathrm{m}\right)$ e frequência em raios/mm, considerando-se 40 repetições.

\subsubsection{Mensuração das fibras}

Em todas os discos do lenho no DAP, as amostras foram retiradas nas posições de transição do cerne/ alburno (cerne periférico), tomando-se os cavacos em todo os plano radial das amostras, que posteriormente foram submetidos ao processo de maceração realizado de acordo com o método do peróxido de hidrogênio/ ácido acético descrito por Ramalho (1987). As mensurações das fibras foram realizadas segundo os procedimentos normativos COPANT, (1974), considerando-se 20 repetições. 


\subsection{Análise estatística}

Além da determinação das estatísticas simples como média, desvio-padrão e coeficiente de variação, foi realizada uma análise de variância dos dados, com a adoção de um delineamento inteiramente ao acaso. Uma vez que o F daANOVA se apresentou significativo, foi empregado o teste de Tukey a 5\% de significância, visando verificar as diferenças, entre as regiões, dos parâmetros anatômicos avaliados.

\section{RESULTADOS}

\subsection{Mensuração de elementos vasculares e parênquima radial}

Os valores médios e os respectivos coeficientes de variação, obtidos para os parâmetros relacionados ao diâmetro e frequência dos vasos da madeira do clone avaliado na idade de 64 meses, estão apresentados na Tabela 2.

Por essa tabela se observa que, de modo geral, os vasos de menores diâmetros tangenciais foram encontrados nas regiões de São Mateus e Aracruz, ou seja, vasos com diâmetros de 91,59 e 95,47 $\mu \mathrm{m}$, respectivamente. Os maiores diâmetros vasculares foram verificados no lenho de árvores que cresceram nas regiões de Domingos Martins e Mutum, cujos valores foram de 103,23 e 103,46 $\mu \mathrm{m}$, respectivamente. Os diâmetros vasculares não diferiram estatisticamente nas árvores que cresceram nas regiões de Alto Rio Novo, Aimorés e Domingos Martins.

A região de São Mateus destacou-se por produzir madeira com maior frequência vascular, diferindo estatisticamente das demais a 5\% de significância. Lenhos com menores frequências vasculares foram encontrados nas regiões de Aimorés e Domingos Martins, porém não diferindo estatisticamente.

Os coeficientes de variação do diâmetro dos vasos variaram entre 18,91-23,05\%, demonstrando mediana variação, e pode-se considerar que o diâmetro dos vasos foi pouco heterogêneo entre as regiões.

Os coeficientes de variação da frequência de vasos variaram de $24,14 \%$ na madeira de São Mateus a 30,67\% naquela da região de Aracruz, considerados medianos a elevados, respectivamente.

\subsection{Mensuração do parênquima radial}

Os valores médios dos parâmetros anatômicos do tecido radial da madeira do clone do híbrido natural de Eucalyptus grandis são apresentados na Tabela 3.

A região de Aimorés apresentou maior altura do parênquima radial de $298,41 \mu \mathrm{m}$ e o menor valor igual a 233,07 $\mu \mathrm{m}$ na região de Mutum, diferindo de todas as outras localidades.

Tabela 2- Valores médios de diâmetro e frequência vasculares de madeira de eucalipto.

Table 2 -Average values of diameter and frequency of eucalypt wood.

\begin{tabular}{lcc}
\hline \multicolumn{1}{c}{ Região } & Diâmetro tangencial $(\mu \mathrm{m})$ & Frequência $\left(\mathrm{vasos} / \mathrm{mm}^{2}\right)$ \\
\hline Aracruz - ES & $95,47 \mathrm{c}^{1}$ & $14,32 \mathrm{~b}$ \\
& $(18,05)(18,91)^{*}$ & $(4,39)(30,67)$ \\
Domingos Martins-ES & $103,23 \mathrm{ab}$ & $13,43 \mathrm{~cd}$ \\
& $(22,35)(21,65)$ & $(3,66)(27,23)$ \\
Mutum - MG & $103,46 \mathrm{a}$ & $13,68 \mathrm{c}$ \\
& $(21,52)(20,80)$ & $(3,73)(27,31)$ \\
São Mateus - ES & $91,59 \mathrm{~d}$ & $16,00 \mathrm{a}$ \\
& $(14,57)(16,35)$ & $(3,93)(24,54)$ \\
Aimorés - MG & $100,69 \mathrm{~b}$ & $13,06 \mathrm{~d}$ \\
& $(17,89)(17,76)$ & $(3,54)(27,13)$ \\
Alto Rio Novo - ES & $100,79 \mathrm{~b}$ & $14,32 \mathrm{~b}$ \\
& $(23,23)(23,05)$ & $(4,01)(28,04)$ \\
\hline Média geral & 99,21 & 14,14 \\
& $(19,60)(19,75)$ & $(3,88)(27,48)$ \\
\hline
\end{tabular}

* Valores entre parênteses correspondem ao desvio-padrão ( $\mu \mathrm{m}$ e vasos $/ \mathrm{mm}^{2}$ ) e coeficiente de variação $(\%)$, respectivamente.

${ }^{1}$ Valores médios dos parâmetros mensurados nos raios da madeira de eucalipto. 
Tabela 3 - Valores médios dos parâmetros mensurados nos raios da madeira de eucalipto.

Table 3 - Average values of the parameters measured in the rays in eucalypt wood.

\begin{tabular}{lcccc}
\hline \multicolumn{1}{c}{ Região } & Altura $(\mu \mathrm{m})$ & Largura $(\mu \mathrm{m})$ & Altura $\left(\mathrm{n}^{\text {o de células })}\right.$ & Frequência $(\mathrm{raios} / \mathrm{mm})$ \\
\hline Aracruz - ES & $239,73 \mathrm{de}^{1}(96,03)$ & $8,48 \mathrm{~b}$ & $13,26 \mathrm{c}$ & $12,27 \mathrm{~d}$ \\
& $(40,06)^{*}$ & $(2,09)(24,67)$ & $(4,96)(37,46)$ & $(1,88)(15,30)$ \\
Domingos Martins- ES & $252,85 \mathrm{~cd}$ & $8,46 \mathrm{~b}$ & $14,85 \mathrm{~b}$ & $12,69 \mathrm{bc}$ \\
& $(120,60)(47,70)$ & $(2,32)(27,44)$ & $(7,46)(50,26)$ & $(1,96)(15,43)$ \\
Mutum-MG & $233,07 \mathrm{e}$ & $8,43 \mathrm{~b}$ & $13,35 \mathrm{c}$ & $12,45 \mathrm{~cd}$ \\
& $(102,78)(44,10)$ & $(1,99)(23,60)$ & $(6,10)(45,73)$ & $(1,82)(14,65)$ \\
São Mateus - ES & $261,55 \mathrm{bc}$ & $7,79 \mathrm{c}$ & $14,53 \mathrm{~b}$ & $13,19 \mathrm{a}$ \\
& $(107,69)(41,17)$ & $(2,05)(26,31)$ & $(6,39)(43,96)$ & $(2,08)(15,76)$ \\
Aimorés-MG & $298,01 \mathrm{a}$ & $7,03 \mathrm{~d}$ & $16,05 \mathrm{a}$ & $12,84 \mathrm{ab}$ \\
& $(166,65)(55,92)$ & $(1,89)(26,96)$ & $(4,62)(28,80)$ & $(4,73)(36,84)$ \\
Alto Rio Novo - ES & $271,41 \mathrm{~b}$ & $9,72 \mathrm{a}$ & $15,92 \mathrm{a}$ & $12,76 \mathrm{bc}$ \\
& $(115,83)(42,68)$ & $(3,50)(35,98)$ & $(5,90)(37,07)$ & $(1,98)(15,54)$ \\
\hline Média Geral & 259,44 & 8,32 & 14,66 & 12,70 \\
& $(118,26)(45,27)$ & $(2,31)(27,49)$ & $(5,90)(40,54)$ & $(2,41)(18,92)$ \\
\hline
\end{tabular}

* Valores entre parênteses correspondem ao desvio-padrão ( $\mu \mathrm{m}, \mathrm{n}^{\circ}$ de células e raios $/ \mathrm{mm}^{2}$ ) e coeficiente de variação (\%), respectivamente.

${ }^{1}$ Valores médios ao longo das colunas seguidos de mesma letra minúscula não diferem estatisticamente entre si, pelo teste de Tukey a $5 \%$ de significância..

Os coeficientes de variação, relativos às mensurações da altura do parênquima radial, variaram de 28,80-50,26\%, considerados elevados e indicativos de grande heterogeneidade nesse lenho relativo a esse parâmetro.

Os coeficientes de variação relativos à largura do parênquima radial variaram de $24,67 \%$ nas madeiras da região de Aracruz até o valor de 35,98\% naquelas da região de Alto Rio Novo, todos considerados medianos a elevados, indicando pouca homogeneidade nesses lenhos relativos às dimensões dos raios.

Relativo à frequência do parênquima radial, a região de São Mateus apresentou, de acordo com a Tabela 3 , valor mais elevado de 13,19 raios/mm. Os menores valores foram nas regiões de Aracruz, com 12,27 raios/ $\mathrm{mm}$ e de Mutum, 12,45raios/mm, não diferenciando estatisticamente a $5 \%$ de significância.

O coeficiente de variação analisado para a frequência do parênquima radial na região de Aimorés foi de $36,84 \%$, considerado elevado e refletindo em grande heterogeneidade desses lenhos relativos à frequência radial. À exceção do valor elevado do coeficiente de variação apresentado na madeira produzida na região de Aracruz, todas as demais regiões produziram madeiras mais homogêneas quanto à frequência radial.
Ao considerar o parâmetro da largura de raio em número de células, todas as regiões se diferenciaram estatisticamente. Também quanto à frequência do parênquima radial, todas as regiões diferiram estatisticamente.

\subsection{Mensuração dos elementos fibrosos}

Os valores médios das dimensões das fibras da madeira de um clone do híbrido natural de Eucalyptus grandis são apresentados na Tabela 4.

A região de Aimorés apresentou maior comprimento, correspondendo a 1.051,92 $\mu \mathrm{m}$, enquanto a região de Domingos Martins, o menor valor $(910,65 \mu \mathrm{m})$.

Os coeficientes de variação do comprimento da fibra, na região de Aimorés, foram iguais a 42,12\%, demonstrando maior heterogeneidade no lenho dessas madeiras quanto ao comprimento de suas fibras. Para as madeiras produzidas nas demais regiões, os valores de coeficientes de variação podem ser considerados baixos, ou seja, variaram de 11,97 a 16,85\% .

Quanto à largura das fibras, destacaram-se as regiões de Aimorés (19,58 $\mu \mathrm{m})$ e Alto Rio Novo $(19,30 \mu \mathrm{m})$ como às que produziram madeiras de fibras mais largas, não diferindo estatisticamente entre elas a 5\% de significância. As fibras de menor largura foram encontradas nas madeiras produzidas na região de Aracruz (16,93 $\mu \mathrm{m})$. 
Tabela 4 - Valores médios dos parâmetros mensurados a partir das fibras da madeira de eucalipto. Table 4 -Average values of the parameters measured of the fibers in eucalypt wood.

\begin{tabular}{lcccc}
\hline \multicolumn{1}{c}{ Região } & Comprimento $(\mu \mathrm{m})$ & Largura da Fibra $(\mu \mathrm{m})$ & Diâmetro do Lume $(\mu \mathrm{m})$ & Espessura de Parede $(\mu \mathrm{m})$ \\
\hline Aracruz - ES & $970,84 \mathrm{~b}^{1}$ & $16,93 \mathrm{e}$ & $11,05 \mathrm{c}$ & $2,94 \mathrm{~d}$ \\
& $(152,26)(15,68)^{*}$ & $(3,34)(19,75)$ & $(3,13)(28,32)$ & $(1,00)(34,14)$ \\
Domingos Martins-ES & $910,65 \mathrm{c}$ & $18,76 \mathrm{bc}$ & $11,00 \mathrm{c}$ & $3,88 \mathrm{a}$ \\
& $(153,45)(16,85)$ & $(3,46)(18,45)$ & $(3,44)(31,24)$ & $(1,14)(29,46)$ \\
Mutum-MG & $924,35 \mathrm{c}$ & $18,51 \mathrm{c}$ & $11,31 \mathrm{bc}$ & $3,60 \mathrm{bc}$ \\
& $(137,19)(14,84)$ & $(3,35)(18,09)$ & $(3,03)(26,79)$ & $(0,81)(22,51)$ \\
São Mateus - ES & $994,47 \mathrm{~b}$ & $17,70 \mathrm{~d}$ & $10,72 \mathrm{c}$ & $3,49 \mathrm{c}$ \\
& $(146,95)(14,78)$ & $(3,44)(19,44)$ & $(3,02)(28,20)$ & $(0,88)(25,14)$ \\
Aimorés - MG & $1051,92 \mathrm{a}$ & $19,58 \mathrm{a}$ & $12,09 \mathrm{a}$ & $3,75 \mathrm{ab}$ \\
& $(443,09)(42,12)$ & $(3,20)(16,34)$ & $(3,15)(26,06)$ & $(0,93)(24,83)$ \\
Alto Rio Novo - ES & $984,81 \mathrm{~b}$ & $19,30 \mathrm{ab}$ & $11,64 \mathrm{ab}$ & $3,83 \mathrm{a}$ \\
& $(117,93)(11,97)$ & $(3,21)(16,61)$ & $(3,00)(25,79)$ & $(0,92)(24,02)$ \\
\hline Média Geral & 972,84 & 18,46 & 11,30 & 3,58 \\
& $(191,81)(19,37)$ & $(3,33)(18,11)$ & $(3,13)(27,73)$ & $(0,95)(26,68)$ \\
\hline
\end{tabular}

* Valores entre parênteses correspondem ao desvio-padrão $(\mu \mathrm{m})$ e coeficiente de variação $(\%)$, respectivamente.

Os coeficientes de variação da largura da fibra, em todas as regiões analisadas, ficaram entre 16-20\%, indicando que o material avaliado possui heterogeneidade mediana relativa à largura das fibras do xilema secundário.

O diâmetro do lume apresentou os maiores valores nas regiões de Aimorés, com 12,09 $\mu$ m e Alto Rio Novo $(11,64 \mu \mathrm{m})$, que não diferiram estatisticamente.

Os valores de coeficiente de variação foram bastante elevados em todas as regiões, relativos ao diâmetro de lume, indicando presença de lenhos bastante heterogêneos no que diz respeito ao lume das fibras.

O coeficiente de variação da espessura de parede variou de 22,51 a 34,14\%, indicando também a formação de lenhos muito heterogêneos em relação a esse parâmetro, em todas as regiões.

\section{DISCUSSÃO}

Alzate (2004), avaliando as propriedades anatômicas da madeira de clones de Eucalyptus grandis com 8 anos de idade que cresceram no Estado de São Paulo, encontrou valor de diâmetro tangencial dos vasos igual a $106,00 \mu \mathrm{m}$. Tal valor é próximo daquele encontrado neste estudo nas regiões de Domingos Martins-ES e Mutum- MG.

Como é de se esperar, as madeiras que apresentaram os menores diâmetros vasculares foram aquelas com maior frequência vascular. Alzate (2004), também em seu estudo sobre a anatomia da madeira de árvores de Eucalyptus grandis com idade de 8 anos, encontrou valores de frequência vascular variando de $9-14$ vasos $/ \mathrm{mm}^{2}$, portanto próxima aos verificados neste estudo em praticamente todas as regiões.

As regiões de Aracruz e São Mateus, de menores altitudes e com maiores precipitações, foram as que produziram as madeiras com menores diâmetros vasculares e, consequentemente, maiores frequências desses elementos.

Comparando a descrição de Alfonso (1987) ao analisar as características anatômicas da madeira de Eucalyptus grandis com a idade de 8 anos, verificaram-se valores da altura do parênquima radial variando entre 120,00-290,00-450,00 $\mu \mathrm{m}$. Alzate (2004), avaliando o clone da madeira de Eucalyptus grandis com a idade de 8 anos, encontrou valores de altura do parênquima radial entre 160,00-180,00 $\mu \mathrm{m}$, considerados bastante inferiores aos obtidos neste trabalho.

O valor médio da largura do parênquima radial observado (Tabela 3 ) na região de Alto Rio Novo foi de $9,72 \mu \mathrm{m}$ e, na região de Aimorés, de 7,03 $\mu \mathrm{m}$, sendo maior e menor, respectivamente. Alfonso (1987), ao descrever as características anatômicas da madeira de Eucalyptus grandis com 8 anos de idade, relatou valores de largura do parênquima radial variando de 8-13-22 $\mu \mathrm{m}$, ligeiramente superiores aos apresentados na Tabela 3.

Revista Árvore, Viçosa-MG, v.36, n.3, p.559-567, 2012 
Alfonso (1987) encontrou valores de frequência de raios variando entre 5-9-14 raios/mm na madeira de Eucalyptus grandis com 8 anos de idade. Quando comparados com os valores obtidos por Alzate (2004) na mesma espécie, foram de 13-16 raios/mm, portanto mais elevados que aqueles obtidos neste estudo.

Alzate (2004), ao avaliar o clone de Eucalyptus grandis com idade de 8 anos, relatou que a média do comprimento das fibras analisadas foi de 1.030,00 $\mu \mathrm{m}$, superior aos valores apresentados na Tabela 4.

Tomazello Filho (1985), ao avaliar as características anatômicas da madeira de Eucalyptus grandis com 10 anos de idade, relatou o comprimento das fibras com o valor médio de 1.200,00 $\mu \mathrm{m}$. Outros autores, como Barrichelo e Brito (1976), ao analisarem as características anatômicas da madeira de Eucalyptus grandis com idade de 5 anos, relataram o comprimento de fibras de $1.030,00 \mu \mathrm{m}$.

Alzate (2004) encontrou valores de largura de fibras de 20,99 $\mu \mathrm{m}$, Tomazello Filho (1985) relatou largura de fibras de 23,00 $\mu \mathrm{m}$ e Barrichelo e Brito (1976) apresentou valor de 20,50 $\mu \mathrm{m}$ para a madeira de Eucalyptus grandis que cresceu no Estado de São Paulo. Esses mesmos autores obtiveram valores de diâmetro de lume de 9,58; 12,20; e 10,30 $\mu \mathrm{m}$, respectivamente, nas madeiras de 8-10 anos que também cresceram em São Paulo.

Analisando a Tabela 4, vê-se que, quanto à espessura da parede, os maiores valores foram encontrados nas regiões de Domingos Martins (3,88 $\mu \mathrm{m})$, Alto Rio Novo (3,83 $\mu \mathrm{m})$ e Aimorés $(3,75 \mu \mathrm{m})$, não se diferindo estatisticamente. As fibras de paredes mais delgadas foram produzidas na madeira da região de Aracruz, caracterizada por baixa altitude (49 m) e maior quantidade de chuva média anual $(1.337 \mathrm{~mm})$ em relação às demais regiões em estudo.

Alzate (2004), Barrichelo e Brito (1976) e Tomazello Filho (1985) encontraram, na mesma espécie deste estudo, valores de espessura de parede de 5,70; 5,10; e $5,40 \mu \mathrm{m}$, respectivamente.

\section{CONCLUSÕES}

Com base nos resultados do estudo de anatomia da madeira de um clone do híbrido natural de Eucalyptus grandis, pode-se concluir que o diâmetro tangencial e a frequência vascular foram variáveis nas diferentes regiões, e nas regiões de baixa altitude e maior precipitação a madeira produzida caracterizou-se por menores diâmetros vasculares e, consequentemente, maiores frequências desses elementos.

Relativo aos raios, também foram verificadas diferenças relativas à altura, largura e frequência nas diversas regiões. Tais diferenças foram acentuadas nos parâmetros relativos a altura e frequência radiais.

Os parâmetros relativos às dimensões das fibras foram variáveis nas diferentes regiões avaliadas. As madeiras produzidas na região de Aracruz possuem fibras de comprimento e espessura de parede muito inferiores às produzidas nas demais regiões estudadas. Tanto a baixa altitude quanto a mais elevada precipitação nessa região favoreceram a formação de fibras de paredes mais delgadas que aquelas formadas no xilema secundário de árvores que cresceram nas demais regiões.

As características anatômicas da madeira foram significativamente variáveis nas diversas regiões, o que demonstra a importância de serem avaliadas quando se busca a qualidade de madeira para a produção de celulose.

\section{AGRADECIMENTOS}

À empresa VCP - Unidade Aracruz, pela parceria com a UFES, que possibilitou a realização deste trabalho; e ao CNPq, pela concessão de bolsa de produtividade em pesquisa ao professor orientador.

\section{REFERÊNCIAS}

ALFONSO, V. A. Caracterização anatômica do lenho e da casca das principais espécies de Eucalyptus L' Herit cultivadas no Brasil. 1987. 188f. Tese (Doutorado em Ciências Biológicas - Botânica) Instituto de Biociências/Universidade de São Paulo, São Paulo, 1987.

AlZATE, S. B. A. Caracterização da madeira de árvores de clones de Eucalyptus grandis, E. saligna e E.grandis $x$ E.urophylla. 2004. 133f. Tese (Doutorado em Recursos Florestais) - Escola Superior de Agricultura Luiz de Queiroz, Universidade Federal de São Paulo, Piracicaba, 2004.

BAMBER, R. K.; HORNE, R.; GRAHAN-HIGGS, A. Effect of fast growth on the wood properties of Eucalyptus grandis. Australian Forest Research, v.12, p.163-167, 1982. 
BAMBER, R. K. The wood anatomy of eucalypts and papermaking. Appita, v.38, n.3, p.210-216, 1985.

BAMBER, R. K.; HUMPHREY, F. R. A preliminary study of some wood properties of Eucalyptus grandis (Hill) Maiden. Journal of the Institute of Wood Science, v.11, n.1, p.66-70, 1963.

BARRICHELO, L. E. G.; BRITO, J. O. A madeira das espécies de eucalipto como matéria-prima para a indústria de celulose e papel. Brasília: PNUD/FAO/IBDF/ BRA-45. PRODEPEF, 1976. 145p. (Série Divulgação, 13).

COPANT - Comission Panamericana de Normas Técnicas Descripción de características generales, macroscópicas de las maderas angiospermas dicotiledóneas. COPANT, v.30, p.1-19, 1974.

DRUMOND, M. A.; OLIVEIRA, V. R.; CARVALHO, O. M. Comportamento silvicultural de espécies e procedências de Eucalyptus na região dos Tabuleiros Costeiros do Estado de Sergipe. Revista Árvore, v.22, n.1, p.137-142, 1998.

DUPLOOY, A. B. J. The relationship between Wood and pulp properties of E. grandis (Hill exMaiden) grown in South Africa. Appita, v.22, n.4, p.257-264, 1980.

GONÇALVES. J. L. M. et al. Silvicultural effects on the productivity and wood quality of eucalypt plantations. Forest Ecology Management, v.193, p.45-61, 2004.

OLIVEIRA, J. T. S. Caracterização da madeira de eucalipto para a construção civil. 1998. 429f. Tese (Doutorado em Engenharia Civil) - Escola Politécnica da Universidade de São Paulo, São Paulo, 1998.

PIRES, C. S. P.; PARENTE, P. R. Competição de espécies de origem de Eucalyptus na região de Mogi Mirin, SP. Silvicultura, v.11, n.41, 1-114, 1986.
RAMALHO, R. S. O uso de macerado no estudo anatômico de madeiras. Viçosa, MG: Universidade Federal de Viçosa, 1987. 4p.

RAMÍREZ, M.; RODRÍGUEZ, J. Wood anatomy and biometric parameters variation of Eucalyptus globulus clones. Wood Science and Technology, v.43, p.131-141, 2009.

RODRIGUES, L. C.; VASTANO JÚNIOR, B.; SILVA, A. P. Manejo e melhoramento de florestas de Eucalyptus em áreas de areias quartzonas na região Nordeste de Estado de São Paulo. Silvicultura, v.11, n.41, p.104$109,1986$.

SILVA, J. C. et al. Influência de idade e da posição radial nas dimensões das fibras e dos vasos da madeira de Eucalyptus grandis Hill ex Maiden Wood. Revista Árvore, v.31, n. 6, p.795-799, 2007.

TOMAZELLO FILHO, M. Variação radial dos constituintes anatômicas e da densidade básica da madeira de oito espécies de eucalipto. Piracicaba: ESALQ/ LCF, 1983. 102p.

TOMAZELLO FILHO, M. Variação radial da densidade básica e da estrutura anatômica da madeira de Eucalyptus saligna e Eucalyptus grandis. IPEF, v.29, p.37-45, 1985.

TRUGILHO, P. F. et al. Classificação de clones de Eucalyptus sp visando à produção de polpa celulósica. Revista

Árvore, v.28, n.6, p.895-899, 2004.

WHER, T. R. Variação nas características da madeira de Eucalyptus grandis Hill ex Maiden e suas influências na qualidade de cavacos em cozimento Kraft. 1991. 84f. Dissertação (Mestrado em Recursos Florestais) - Escola Superior de Agricultura "Luiz de Queiroz", Universidade de São Paulo, Piracicaba, 1991. 